University of Nebraska - Lincoln

DigitalCommons@University of Nebraska - Lincoln

May 2000

\title{
Curie temperature of multiphase nanostructures
}

\author{
Ralph Skomski \\ University of Nebraska-Lincoln, rskomski2@unl.edu \\ David J. Sellmyer \\ University of Nebraska-Lincoln, dsellmyer@unl.edu
}

Follow this and additional works at: https://digitalcommons.unl.edu/physicssellmyer

Part of the Physics Commons

Skomski, Ralph and Sellmyer, David J., "Curie temperature of multiphase nanostructures" (2000). David Sellmyer Publications. 66.

https://digitalcommons.unl.edu/physicssellmyer/66

This Article is brought to you for free and open access by the Research Papers in Physics and Astronomy at DigitalCommons@University of Nebraska - Lincoln. It has been accepted for inclusion in David Sellmyer Publications by an authorized administrator of DigitalCommons@University of Nebraska - Lincoln. 


\title{
Curie temperature of multiphase nanostructures
}

\author{
R. Skomskia) and D. J. Sellmyer \\ Department of Physics and Astronomy and Center for Materials Research and Analysis, \\ University of Nebraska, Lincoln, Nebraska 68588
}

The Curie temperature and the local spontaneous magnetization of ferromagnetic nanocomposites are investigated. The macroscopic character of the critical fluctuations responsible for the onset of ferromagnetic order means that there is only one Curie temperature, independent of the number of magnetic phases present. The Curie temperature increases with the grain size and is, in general, larger than predicted from the volume averages of the exchange constants. However, the Curie-temperature enhancement is accompanied by a relative reduction of the spontaneous magnetization. Due to the quadratic dependence of the permanent-magnet energy product on the spontaneous magnetization, this amounts to a deterioration of the magnets performance. The length scale on which an effective intergranular exchange coupling is realized (coupling length) depends on the Curie-temperature difference between the phases and on the spacial distribution of the local interatomic exchange. As a rule, it is of the order of a few interatomic distances; for much bigger grain sizes the structures mimic an interaction-free ensemble of different ferromagnetic materials. This must be compared to the magnetic-anisotropy coupling length, which is of the order of $10 \mathrm{~nm}$. The difference is explained by the nonrelativistic character of the Curie-temperature problem. (C) 2000 American Institute of Physics. [S0021-8979(00)63608-3]

\section{INTRODUCTION}

The onset of long-range order in ferromagnets has been a long-standing and mind-stimulating problem. Since the first third of the 20th century, when research focussed on the basic relation between quantum mechanics and magnetic order, ${ }^{1,2}$ interest has shifted towards critical phenomena ${ }^{3,4}$ and the behavior of particular magnetic features such as surfaces ${ }^{5,6}$ and artificial structures. ${ }^{7-13}$ In the case of multilayers, mean-field Curie temperatures are obtained by diagonalizing tridiagonal exchange-interaction matrices, ${ }^{7-10}$ whereas Wang and Mills ${ }^{11}$ used a Landau-Ginzburg type analysis to elaborate that the spontaneous magnetization varies continuously across phase boundaries.

A problem of scientific and technological interest is the Curie-temperature and spontaneous-magnetization behavior of nanocomposites. There are attempts to use the concept of exchange-field penetration known from multilayers to construct effective coupling parameters, ${ }^{14}$ but there is no comprehensive theoretical understanding of nanocrystalline alloys. A particular question, important in the context of hightemperature permanent magnetism, is whether the Curie temperature $T_{L}$ of any magnetic material can be improved by nanoscale exchange coupling to a phase having a Curie temperature $T_{H}>T_{L}$. In the context of magnetic hysteresis, it has been shown that interatomic exchange can be exploited to improve the hard-magnetic performance of a magnet by adding soft-magnetic material. ${ }^{15,16}$ In that case, the coupling between the hard and soft regions is very effective on length scales up to about $10 \mathrm{~nm}$.

\footnotetext{
${ }^{a)}$ Electronic mail: rskomski@unlserve.unl.edu
}

\section{CALCULATION AND RESULTS}

\section{A. Curie temperature and spontaneous magnetization}

Let us start with a mean-field analysis of ferromagnetic nanocomposites. By definition, the spontaneous magnetization vanishes at the Curie temperature, so that the mean-field equations can be linearized and the spontaneous magnetization $M_{i}=M_{z}\left(\mathbf{r}_{i}\right)$ of the $i$ th atom obeys

$$
T M_{i}=\frac{1}{k_{B}} \sum_{j} J_{i j} M_{j}
$$

In this equation, the $J_{i j}=J\left(\mathbf{r}_{i}-\mathbf{r}_{J}\right)$ are local exchange parameters whose detailed meaning depends on the underlying model. Diagonalization of Eq. (1) yields a common Curie temperature, which is equal to the highest eigenvalue of the matrix $J_{i j} / k_{B}$. For multilayers, it has been found that the Curie temperature increases with increasing layer periodicity. ${ }^{7,9}$ In the case of three-dimensional nanostructures, of arbitrary geometry, the matrix $J_{i j} / k_{B}$ is no longer tridiagonal and the diagonalization is slightly more complicated. Figure 1 shows cubic inclusions of a material with a high (bulk) Curie temperature $T_{H}$ in a matrix of a material having a low (bulk) Curie temperature $T_{L}<T_{H}$. The bars show the Curie temperatures for $T_{H}=1000 \mathrm{~K}, T_{L}=400 \mathrm{~K}$, and an intermediate nearest-neighbor interface exchange of $J_{i j} / k_{B}=700 / 6$. As in the case of multilayers, $T_{C}$ increases with the size of the $T_{H}$ and $T_{L}$ regions, and the composite Curie temperature is close to $T_{H}$.

The eigenmode belonging to $T_{C}$ gives the spacial variation of the order parameter in the vicinity of $T_{C}$. [The other eigenvalues and eigenmodes of Eq. (1) have no physical meaning, because the spontaneous magnetization is finite below $T_{C}$ and the mean-field equations are no longer linear.] Below $T_{C}$, the Brillouin-type nonlinearity of the mean-field 

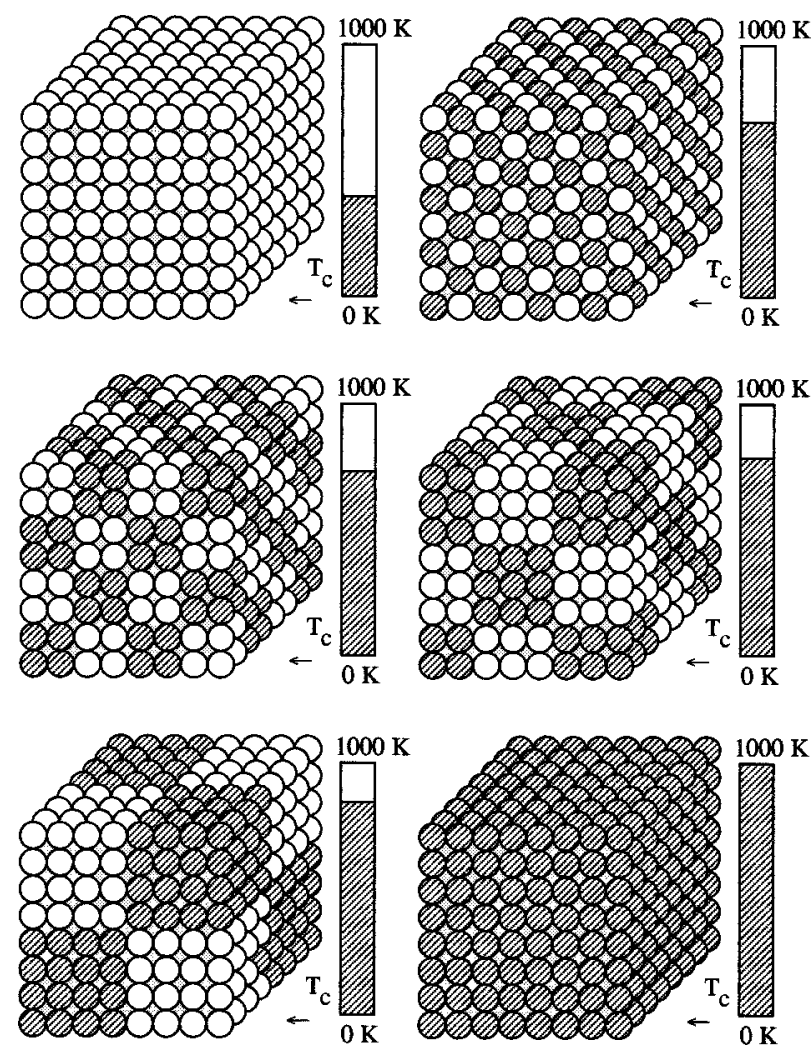

FIG. 1. Mean-field Curie temperatures of three-dimensional composites. The dark and bright areas correspond to high and low bulk Curie temperatures $T_{H}=1000 \mathrm{~K}$ and $T_{L}=400 \mathrm{~K}$, respectively.

equations is important. For $N$ nonequivalent sites, the local spontaneous magnetization $M_{i}(T)$ is obtained by solving the set of $N$ nonlinear mean-field equations. Figure 2 shows a typical result for $N=4$ and classical $(J=\infty)$ Heisenberg spins. The considered structure exhibits four nonequivalent sites, one $T_{H}$ site and three $T_{L}$ sites, and each nonequivalent site has a different spontaneous magnetization below $T_{C}$. In

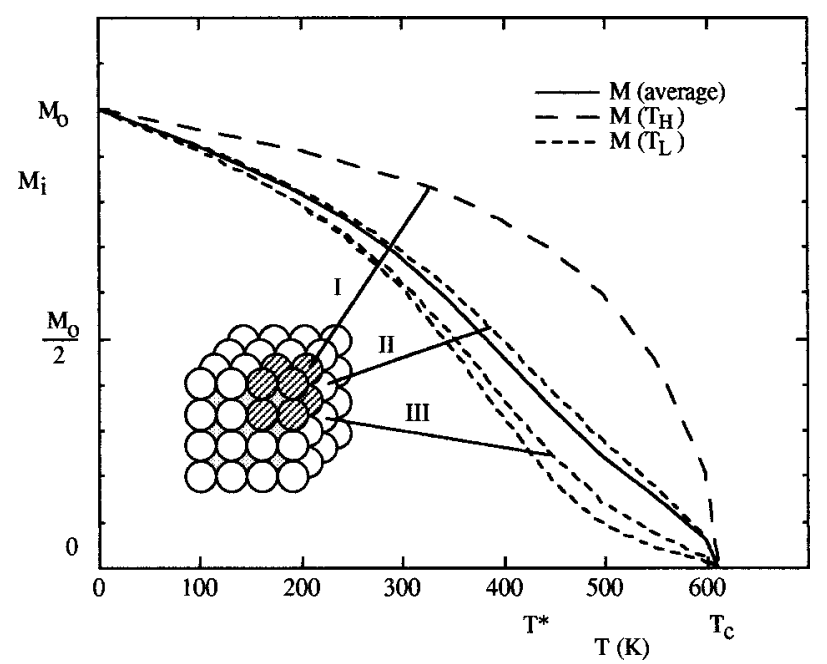

FIG. 2. Temperature dependence of the local spontaneous magnetization (classical Heisenberg model). There are four nonequivalent sites: one $T_{H}$ site and three $T_{L}$ sites. The $T_{L}$ are classified by their position relative to the $T_{H}$ site: nearest neighbors, neighbors connected by face diagonals, and neighbors connected by cube diagonals. The Curie temperature is $611.9 \mathrm{~K}$. the $T_{L}$ phase, the spontaneous magnetization is largest for sites which are nearest neighbors to $T_{H}$ sites. This is related to the above-mentioned continuity of the order parameter at phase boundaries and shows that the spontaneous magnetization is not element specific but depends on the distance from the $T_{H}-T_{L}$ interfaces.

The lower single-phase Curie temperature $T_{L}$ does not translate into any phase transition, although there are resonance-like magnetization features at some nonsingular temperature $T^{*} \approx T_{L}$ (Fig. 2). This is reminiscent of the many-sublattice behavior of garnets and rare-earth transitionmetal intermetallics: ${ }^{12,16,17}$ the rare-earth (or " $T_{L}$ ") atoms are subject to the quasiparamagnetic exchange field of the iron-series (or " $T_{H}$ ") atoms.

Above the thermodynamically not very well defined temperature $T^{*}$, the spontaneous magnetization for $T_{L}$ sites far away from $T_{H}$ sites is very low, but it remains nonzero between $T^{*}$ and $T_{C}$. This smallness of the $T_{L}$ magnetization is of practical importance. In particular, the quadratic dependence of the permanent-magnet energy product on the spontaneous magnetization ${ }^{15,16}$ means that the exchange coupling between $T_{H}$ and $T_{L}$ nanocrystallites is not accompanied by an improvement of the material's permanent magnetic performance. In fact, the $T_{H}$ phase dilutes the magnet's magnetization and reduces the energy product.

\section{B. Range of exchange coupling}

To analyze the coupling between $T_{H}$ and $T_{L}$ regions we transform Eq. (1) into a partial differential equation. Expanding $M_{j}=M_{z}\left[\mathbf{r}_{i}+\left(\mathbf{r}_{j}-\mathbf{r}_{i}\right)\right]$ in terms of $\mathbf{r}_{j}-\mathbf{r}_{i}$ and taking into account that $\left|\mathbf{r}_{j}-\mathbf{r}_{i}\right|=a$ for simple-cubic lattices with nearest-neighbor interactions we obtain after short calculation

$$
\frac{a^{2}}{z} \nabla\left[T_{o}(\mathbf{r}) \nabla M_{z}\right]+\left[T_{o}(\mathbf{r})-T\right] M_{z}=0 .
$$

Here $T_{o}(\mathbf{r})=z J_{o}(\mathbf{r}) / k_{B}$ is a local Curie-temperature parameter so that $T_{o}(\mathbf{r})=T_{L}$ and $T_{o}(\mathbf{r})=T_{H}$ in the $T_{L}$ and $T_{H}$ phases, respectively. For homogeneous magnets, $M_{z}(\mathbf{r})$ $=M_{s}$ and $T_{C}=T_{o}=T_{L}=T_{H}$, but in general Eq. (2) has to be interpreted as a generalized Landau-Ginzburg equation. It differs from the corresponding expression in Ref. 11 by containing the term $\nabla\left[T_{o}(\mathbf{r}) \nabla M_{z}\right]$ rather than the $\nabla^{2} M_{z}$ Landau-Ginzburg term. As analyzed in the context of twophase micromagnetism, ${ }^{15}$ the main effect of the inhomogeneous differential operator is to make the gradient of the eigenfunction discontinuous at phase boundaries. This modifies $T_{C}$ but leaves the behavior of the spontaneous magnetization in a given phase unchanged.

Let us now consider the spontaneous magnetization in the $T_{L}$ phase. The interesting temperature region is between $T^{*}$ and $T_{C}$, because above $T_{C}$ there is no ferromagnetism and below $T_{L}$ the spontaneous magnetization is close to saturation throughout the magnet. We ask to what extent the $T_{H}$ phase is able to create an appreciable spontaneous magnetization in the $T_{L}$ phase. In the considered regime, Eq. (2) yields correlations which decay exponentially with the decay length (correlation length) 


$$
\xi_{L}=\frac{a}{\sqrt{z}} \sqrt{\frac{T_{L}}{T-T_{L}}} .
$$

The correlation length diverges at $T_{L}$, but it decreases with increasing temperature and is of the order of $(a / \sqrt{z}) \sqrt{T_{L} /\left(T_{H}-T_{L}\right)}$ in the vicinity of the Curie temperature. In other words, the coupling or "penetration" length is large when $T_{H} \approx T_{L}$ but does not exceed a few interatomic distances for $T_{L}<T_{H} \approx T_{C}$, that is for materials of interest in two-phase permanent magnetism. The exponential decay of the spontaneous magnetization $M_{z}(\mathbf{r})$ in $T_{L}$ regions quantifies our finding that $M_{z}(r)$ is very small in the centers of extended $T_{L}$ regions.

This scenario is not restricted to the mean-field approximation. Due to renormalization-group effects it is necessary to replace Eq. (3) in the vicinity of $T_{L}$ by ${ }^{18}$

$$
\xi_{L} \approx a\left(\frac{T_{L}}{T-T_{L}}\right)^{\nu},
$$

where $\nu$ is equal to 0.63 and 0.71 for the three-dimensional Ising and Heisenberg models, respectively. However, in the present context Eq. (4) yields only minor corrections to Eq. (3). For example, ${ }^{18}$ grain radii $R$ of order $5 \mathrm{~nm}$ give rise to an "effective Curie-temperature enhancement" of about $1 \%$.

\section{DISCUSSION}

The spacial variation of the spontaneous magnetization and the existence of a unique Curie temperature $T_{C}$ mean that there are neither element-specific magnetization curves in inhomogeneous structures nor order parameters vanishing at different temperatures. However, depending on the phases and coupling mechanism involved, the site-dependent average spontaneous magnetization of the $T_{L}$ phase may be virtually undetectable. ${ }^{13}$ In that case, an exteremely high temperature resolution is necessary to distinguish a composite structure from a superposition of single-phase structures.

It is important to emphasize that Eq. (1) is model independent, that is for appropriately rescaled exchange constants it applies equally well to, e.g., Ising and Heisenberg models. By contrast, due to the nontrivial involvement of the Brillouin functions, the temperature dependence of the local spontaneous magnetization is lattice- and model-dependent. ${ }^{6}$ However, this does not affect the characteristic involvement of the different nonequivalent sites shown in Fig. 2. ${ }^{18}$

Aside from the secondary dependence on $T-T_{L}$, the coupling length scales as the interatomic distance, that is ultimately as the Bohr radius $a_{0}=0.529 \AA$. This must be compared with the "micromagnetic" coupling length (exchange length) $a_{0} / \alpha=7.52 \mathrm{~nm}$, where $\alpha=1 / 137$ is Sommerfeld's fine structure constant. ${ }^{19}$ To explain this difference we note that the inhomogenity $T_{o}(\mathbf{r})-T$ in Eq. (2) is analog to the inhomogenity term $2 K_{1}(\mathbf{r})-\mu_{0} H_{N} M_{s}$ in Ref. 15 . However, anisotropies $\left(K_{1}\right)$ and nucleation fields (coercivities) $H_{N}$ are small relativistic corrections to the leading Coulomb and exchange energies. (In temperature units, they are of the order of $1 \mathrm{~K}$, that is much smaller than $T_{C} \cdot{ }^{16}$ ) Since both the micromagnetic coupling in Ref. 15 and the present "Curietemperature" interaction are realized by interatomic exchange, Curie-temperature disorder is much more effective in reducing the magnetic order than anisotropy disorder.

\section{CONCLUSIONS}

In nanocomposites, there is only one Curie temperature $T_{C}$, independent of the bulk Curie temperatures of phases involved. The Curie temperature is higher than the volume average of the Curie temperatures of the individual phases. Below $T_{C}$, the spontaneous magnetization is site dependent but nonzero throughout the magnet, because regions with strong exchange coupling polarize all other sites. However, in the middle of extended regions with low single-phase $\mathrm{Cu}$ rie temperatures the spontaneous magnetization may be very low, and for large crystallite sizes the overall behavior of the magnet is reminiscent of an interaction-free ensemble of two (or more) ferromagnets with two (or more) Curie temperatures. Due to the nonrelativistic character of the interatomic exchange, the Curie-temperature coupling range is atomic rather than nanoscale, so that the comparatively high Curie temperatures of nanocomposites do not translate into an enhanced permanent-magnet energy product.

\section{ACKNOWLEDGMENT}

This work is supported by DOE-DE-FG-03-98ER45703.

${ }^{1}$ W. Heisenberg, Z. Phys. 49, 619 (1928).

${ }^{2}$ E. Ising, Z. Phys. 31, 253 (1925)

${ }^{3}$ L. Onsager, Phys. Rev. 65, 117 (1944).

${ }^{4}$ K. G. Wilson, Rev. Mod. Phys. 55, 583 (1983).

${ }^{5}$ K. Binder and P. C. Hohenberg, Phys. Rev. B 9, 2194 (1974).

${ }^{6}$ R. Skomski, C. Waldfried, and P. A. Dowben, J. Phys.: Condens. Matter 10, 5833 (1998).

${ }^{7}$ H.-R. Ma and Ch.-H. Tsai, Solid State Commun. 55, 499 (1985).

${ }^{8}$ H. K. Sy, Phys. Lett. A 120, 203 (1987).

${ }^{9}$ W. Maciejewski and A. Duda, Solid State Commun. 64, 557 (1987).

${ }^{10}$ W. Maciejewski, IEEE Trans. Magn. 26, 213 (1990).

${ }^{11}$ R. W. Wang and D. L. Mills, Phys. Rev. B 46, 11681 (1992).

${ }^{12}$ R. Skomski, J. Appl. Phys. 83, 6724 (1998).

${ }^{13}$ U. Bovensiepen, F. Wilhelm, P. Srivastava, P. Poulopoulos, M. Farle, A. Ney, and K. Baberschke, Phys. Rev. Lett. 81, 2368 (1998).

${ }^{14}$ I. Navarro, M. Ortuño, and A. Hermando, Phys. Rev. B 53, 11656 (1996).

${ }^{15}$ R. Skomski and J. M. D. Coey, Phys. Rev. B 48, 15812 (1993).

${ }^{16}$ R. Skomski and J. M. D. Coey, Permanent Magnetism (Institute of Physics, Bristol, 1999).

${ }^{17}$ A. Herpin, Théorie du Magnétisme (Institut National des Sciences et Techniques Nucléaires, Saclay, 1968).

${ }^{18}$ R. Skomski (unpublished).

${ }^{19}$ R. Skomski, H.-P. Oepen, and J. Kirschner, Phys. Rev. B 58, 3223 (1998). 